Las prácticas contables, la evolución informática, su enseñanza y la aplicación de métodos de aula activa en la Contaduría.

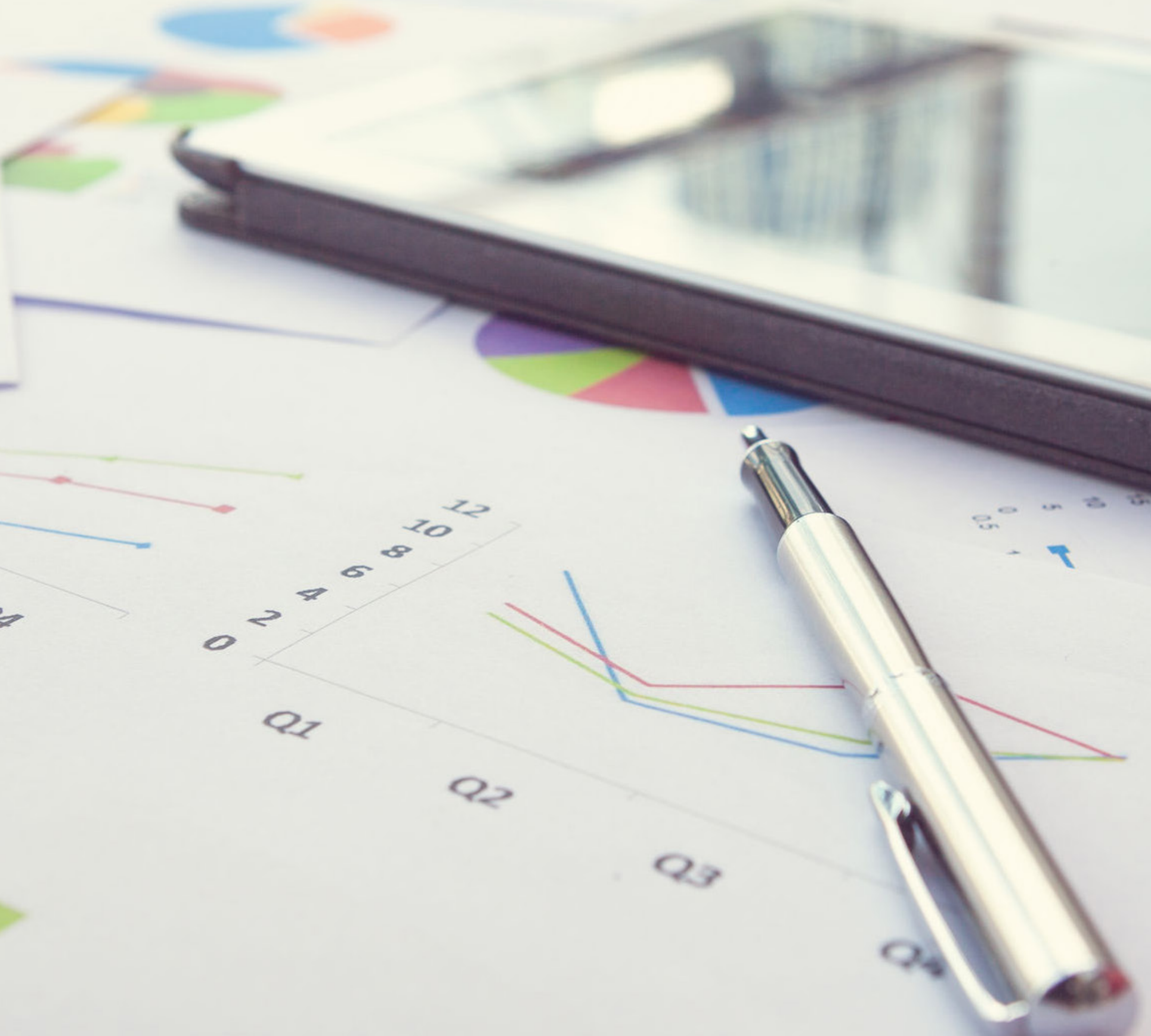




\title{
Las prácticas contables, la evolución informática, su enseñanza y la aplicación de métodos de aula activa en la Contaduría.
}

\author{
Lic. Jorge Araya Sánchez \\ Escuela de Contaduría Pública \\ Universidad Fidélitas \\ San José, Costa Rica \\ joaraya@ufidelitas.ac.cr
}

\begin{abstract}
The purpose of this article is to establish the evolution that accounting systems have had respect to the internal computing automation processes of companies, the reality of the world in the face of the fourth industrial revolution, globalization and the effect of data in our profession. It will cover the momentum that has developed our country with the imposition of the electronic invoicing system and other tax changes. As another important element, we will cover the need to take the teaching of Accounting to areas where the conjunction of formal systems of International Standards converges in the application of the daily "practice" of the Accountant's activity.
\end{abstract}

We will make an effort to propose an example of a theoreticalpractical topic, such as the preparation of the fourth Basic Financial Statement of accounting, where the technique of the flipped classroom, the resolution of cases and critical thinking are combined in the solution of the proposed technical topic.

Key Words: Accounting, Computing, Tax, NIFF, Active Learning.

\section{Introducción:}

Entender la evolución de la práctica contable implica mirar detenidamente el entorno de los negocios en el mundo, son muchos los elementos que han caracterizado nuestra historia en los últimos años, pero estamos convencidos que en la materia que nos aplica, son dos elementos de mayor influencia en estas prácticas y producto de la tercera revolución industrial vivida en la segunda mitad del siglo anterior:

La automatización de los procesos internos de las empresas gracias al vertiginoso crecimiento de la informática.

La globalización mundial del comercio y de la industria, que ha empujado hacia la uniformidad y control de los negocios internacionales, por supuesto ha implicado la unificación de las prácticas contables y la adopción de normas estándares de interpretación de las transacciones contables que generan los procesos empresariales de dichas empresas globales y nacionales.

Nuestro país no ha escapado a este contexto y por el contrario existen actores en el área contable que han buscado que estos dos puntos de influencia empiecen a implementarse en la realidad de nuestras empresas, los Colegios de Contadores, tanto público como privado han hecho por más de 20 años este gran esfuerzo por capacitar y actualizar a los profesionales en los campos de aplicación de las Normas Internacionales de Información Financiera y la utilización de herramientas contables en las empresas de cualquier sector productivo.

Coherentemente a esta realidad sobresale el papel que ha realizado el Estado, específicamente el Ministerio de Hacienda de Costa Rica, en los tres últimos años, el cual con un grado de presura sobre los empresarios ha impuesto el modelo de Facturación Electrónica vigente a la fecha, la adopción de la normativa internacional y nuevos modelos impositivos que transforman el fondo de comercio de algunas actividades, y su senda influencia en la promoción de un indudablemente cambio en las prácticas contables tradicionales, que nuestras empresas están en compromiso de superar, acompañados de Contadores Públicos y Privados más actualizados a la realidad que se nos avecina y acompaña.

\section{Marco Metodológico:}

La propuesta actual de este artículo es realizar un proceso descriptivo e investigativo que busque como objetivos:

- Entender los principales cambios que la realidad de las empresas y del mundo ha generado en los sistemas contables. 
- Justificar la necesidad que los sistemas educativos en torno a la Contabilidad Moderna se adapten a los cambios en el mundo de los negocios.

- Realizar una propuesta específica ya probada en un aula de la Universidad Fidélitas que permita marcar el futuro de los cambios que los profesores de esta carrera podemos realizar en nuestras aulas.

\section{Marco Teórico}

\section{El cambio en el mundo de los Negocios: La Tercera Revolución Industrial.}

La Revolución Tecnológica y la Contabilidad: Es indudable que nuestros tiempos han evolucionado cada vez con más prisa y presión, lo que hace 20 años era un horizonte de largo plazo, ahora en nuestras realidades actuales en un quinquenio o menos, nos encontramos ante sorprendentes cambios, que no solo afectan directamente a las empresas y negocios, sino muy profundamente a la población y los consumidores.

El desarrollo de la informática que se ha caracterizado por:

- Procesos de mayor velocidad.

- Aumento en la capacidad de manipular datos.

- Aparatos más pequeños pero más poderosos.

- La interconectividad inter-empresa y el internet y la nube.

- Ha llegado directamente al usuario y / o consumidor.

Es indudable que estos cinco motores hayan influenciado cambios en las prácticas empresariales e indudablemente en la gestión contable de estas, las cuales en mayor grado de relevancia encontramos:

- La simplificación de los procesos operativos y del registro contable.

- La generación del comprobante contable en la actividad fuente o documento de negocio que genera la transacción.

- La automatización de los sistemas contables, integrados a las funciones auxiliares más importantes del negocio, ventas, cobro, inventario, cuentas por pagar, bancos y otros, que como señalamos anteriormente, generan los datos para la preparación de la información contable.

- El archivo de datos más accesible y fácil.

- La generación de Estados Financieros completos, comparativos y justificados con información cierta $\mathrm{y}$ válida para cada área de desempeño operativo.

- Ha facilitado el control interno y la capacidad de verificación, auditoría y emisión de opinión sobre los Estados Financieros por parte del Contador Público.
Es imprescindible señalar que esta revolución ha traído consigo el cambio del papel del Contador hacia la Administración de la Tecnología Contable, la distribución de las responsabilidades de supervisión de las fuentes de datos y su capacidad de ser verificada a través de documentos de carácter físico que permitan comprobar uno de los principales exigencias de nuestro sistema contable, el principio de materialidad.

De ahí se ha dado constantes cambios de algunas empresas a la búsqueda de sistemas cada vez más robustos y probados que satisfagan las necesidades operativas de las mismas y la consecuente administración de la información contable que se requiere para administrar el negocio. Al no ser nuestro objeto de estudio los diferentes sistemas de información que ofrece el mercado, tanto de aplicaciones a la medida, desarrollo propio como sistemas de bajo, medio y alto nivel, partiremos de la premisa que dicha escogencia se ha basado en costos de oportunidad funcional y económica que justifican claramente dicha selección.

El énfasis en esta etapa recae en que la contabilidad se caracteriza por ser un proceso ordenado de clasificación de información y resguardo de documentación física que cumpla como comprobante de la materialidad (existencia) de los mismos, dando gran cantidad de trabajo al contador en el archivo, resguardo y protección de dicha información.

Una de las contribuciones más importantes que trajo esta etapa de la evolución contable, es que permitió al contador dedicar una gran cantidad de su tiempo en lugar del registro manual de las transacciones a la revisión, verificación de los procesos y al análisis de las información contable automatizada generada, su comparación analítica y toma de decisiones sobre el negocio.

La Globalización Mundial y las Normas de Información Financiera: A medida que el mundo informático se ha desarrollado, el mundo de los negocios globales ha impactado fuertemente nuevos modelos de prácticas comerciales y empresariales que han impactado fuertemente sobre las prácticas de la contabilidad.

Como parte de este nuevo esquema de negocios mundiales surge la necesidad de contar con sistemas globales de control de la calidad de la información contable, que permita que el comercio fluya entre los países, con información de los diferentes actores (Estados Financieros) basados en normas y valores contables de igualdad interpretativa, uniformidad, fiabilidad, comparabilidad y presentación estandarizada de la información contable.

Estamos hoy en un mundo presente y en nuestra realidad contable que el Contador debe conocer y dominar el cuerpo normativo internacional, llamado NIIF, Normas Internacionales de Información Financiera, [1] promulgadas, acogidas y aceptados por los diferentes actores de la realidad contable costarricense. 
No se puede negar por tanto que este basto mundo de Normas Internacionales que acuerpan 25 NICS y 17 NIIFS vigentes, son de alto impacto no solo en el ejercicio de la profesión contable sino en la enseñanza misma de la Contabilidad.

En nuestro ejercicio de aplicación desarrollamos un caso basado en la aplicación de técnicas de enseñanza activa o STEM, donde tendremos la oportunidad de ayudar al estudiante en la comprensión e interpretación de la normativa específica que abarca este tema, a través del estudio de dos capítulos de libros complementarios del tema.

\section{El papel del Gobierno, factura electrónica, la recaudación de impuestos y algunos cambios en las prácticas contables en el año 2019 en Costa Rica.}

Estamos claramente en un proceso de convencimiento que las prácticas contables modernas han evolucionado, se han visto influenciado por el avance tecnológico y la globalización a través de las normas internacionales, pero quedaría este ambiente incompleto si no hacemos referencia a otro elemento actual, la materia fiscal, que ha realizado una presión a fuertes cambios en las prácticas contables tradicionales de los contadores.

Es indudable que se han presentado tres elementos que en el año presente han impactado fuertemente en las prácticas contables, revisemos el impacto en las mismas:

- La puesta en marcha del sistema de facturación electrónico. El elemento fundamental del sistema es que, aparte de requerir que todo documento de venta, compra, nota de crédito o débito que circule en el sistema sea aceptada por el receptor o sea validada para efectos fiscales, nos ha permitido generar bases de datos de las compras y gastos que deben entrar a los sistemas de información interna. Este elemento permite que la comprobación de datos validados, (materialidad) sea la que el contador utilice, no solo para efectos fiscales sino contables del sistema interior de datos de la empresa. Por otro lado se elimina el documento en físico o papel como comprobante material de las transacciones, disminuyendo considerablemente los archivos de documentos contables, pero obligando a los contribuyentes y contadores a resguardar la información electrónica de la factura en PDF y su comprobante xml en archivos electrónicos controlados, resguardados y respaldados.

- La adopción de las Normas Internacionales de Información Financiera (NIIF) por parte del Gobierno como método de contabilización contable. En junio del 2018 la Dirección General de Tributación emitió la resolución DGT-029 en la cual establece los mecanismos de adopción obligatoria para las empresas de las NIIF. [2] Salvo el método de valoración a valor razonable, la Administración Tributaria ha indicado que todos los mecanismos establecidos en las NIIF son de acatamiento obligatorio para todas las empresas. El efecto más inmediato de esto se da en que las empresas deben preparar adicionalmente al Balance General y el Estado de Resultados, el Estado de Cambios al Patrimonio y el Flujo de Efectivo, adicionando las notas detalladas al cuerpo de Estados Financieros. De hecho los bancos para efectos de trámites crediticios están solicitando el cumplimiento de este precepto, incluyendo en las notas una declaración del Contador que los Estados están de acuerdo a las NIIF.

- Los cambios en algunos regímenes de negocios en las formas impositivas y su efecto sobre las prácticas contables. En este apartado nos encontramos a los cambios impositivos de las Ley 9635 de Fortalecimiento de las Finanzas Públicas, [3] la cual realizó cambios impositivos a segmentos de negocio como rentas de capital inmobiliario y mobiliario para liquidar sus rentas en forma única y mensual, lo que implica un cambio en la práctica contable que implicará una conciliación fiscal entre la contabilidad real de la empresa y las rentas cobradas en forma mensual.

\section{La Contabilidad bajo enseñanza activa.}

Uno de los enfoques presentes de este artículo es que la evolución de los procesos contables se ha visto impactado por el desarrollo informático, el mundo globalizado y el papel que ha tenido el gobierno en nuevas prácticas fiscales que han impulsado un cambio en los formas de procesamiento, interpretación y presentación de información contable económica y financiera.

No es nuestro objeto definir al contador del futuro, hago mías las palabras de la Lic. Dorys López, “ El profesional 2030, el que desea mantenerse o incursionar en las ciencias contables requiere fortalezas en formación de análisis de datos, modelos matemáticos y estadísticos, inteligencia artificial y habilidades cognitivas ya que estando en medio de la cuarta revolución industrial indudablemente nuestro ejercicio profesional requiere de nuevas habilidades ante la urgencia para tomar decisiones sobre la información que preparamos o auditamos, situación que tiene un alto impacto en la profesión." [4] Como profesionales contables y docentes tenemos que entender que ya en nuestras aulas nos encontramos con estudiantes que quieren cambiar su rol tradicional de aprendizaje de la repetición y la memoria al entendimiento de los nuevos modelos contables que las empresas exigen, tanto producto de la automatización, la globalización y las NIIF y las nuevas exigencias tributarias.

Si bien la contabilidad como una técnica estándar ha sobrevivido más de 500 años y seguirá siendo un modelo de registro y acumulación de partida doble, la realidad del entorno que hemos analizado anteriormente genera una nueva presión sobre el papel de la educación contable, la pregunta que como contadores y educares nos debemos hacer: ¿Cómo hacer que la educación contable se adapte a este nuevo entorno? 
Si pensáramos en un modelo ideal que permita responder este cuestionamiento actual, tenemos que iniciar un proceso de análisis, pero es importante aterrizar una pronta solución, por tanto, es imprescindible iniciar estableciendo cuáles son los retos y objetivos que se imponen no solo a la profesión contable, si no a su enseñanza:

- La incorporación de la tecnología en el aula.

- El reconocimiento que la técnica contable debe ir acompañada con la utilización de las NIIF para efectos de interpretación de las transacciones contables.

- La utilización de técnicas de estudio que favorezcan la tropicalización de la normativa contable, ante su grado de dificultad, tal y como señala Vernor Mesén, "Lograr que el lector obtenga una mayor y mejor comprensión de los criterios técnicos esbozados por las NIIF, las cuales se caracterizan por estándares de contabilidad de muy alto nivel técnico pero a las vez constituyen reglas de contabilización sumamente complejas, tanto en su desarrollo programático, como en su redacción." [5]

- La incorporación en las aulas de los preceptos fiscales vigentes en todas las áreas y transacciones de la empresa.

- Aplicación de técnicas activas dentro y fuera del aula, que permitan al estudiante empoderarse del aprendizaje. Lograr que los estudiantes puedan tomar el pensamiento crítico en sus manos y puedan con diferentes metodologías, sea casos, "e-learnig", aula invertida, diseño de cosas, proyectos, etc. ser creadores de su propio aprendizaje y que el aula se convierta en un laboratorio capaz de lograr la experiencia a partir de la prueba y el error.

Es indudable que el profesional en la enseñanza contable que demanda nuestra juventud de hoy es y será un actor proactivo de la práctica contable, le corresponderá su capacitación y rompimiento de los métodos tradicionales para el logro de profesionales que sean capaces de incorporarse no solo a nuestros mercados nacionales sino internacionales de trabajo, con una proyección amplia y globalizada.

Dicho proceso será un reto no sólo cultural sino un proceso de cambio de los paradigmas educativos que ha tenido nuestra profesión, tanto el estudiante como el profesor tendrán que dejar sus presentaciones y prácticas tradicionales por la incorporación de conocimientos activos que estimulen el pensamiento crítico, experimental y que fortalezcan en el estudiante aquellas habilidades blandas necesarias para enfrentar la era de la inteligencia artificial y el "big-data" producto de la recién iniciada cuarta revolución industrial.

\section{Aplicación práctica modelo STEM a un caso contable: enseñanza en la preparación del flujo de efectivo.}

El aprendizaje activo parte del acuerdo entre el alumno y el profesor, es una modificación del contrato académico entre ambos, lo que se trata es de crear un ambiente que contemple a los actores del sistema educativo y para lograr ese cambio hay que lograr algunos cambios en el aula:

- Crear un sistema de valores del curso que determinen desde el inicio cuáles son los énfasis del mismo, las reglas y lo que se espera del estudiante ante una nueva actitud hacia el aprendizaje. No se puede ser activo si las condiciones metodológicas del proceso NO están claramente definidas entre sus actores.

- Investigar, determinar y seleccionar cuáles temas de la materia contable que es parte del programa del curso, son susceptibles de aplicar una técnica activa u otra.

- Preparar para esa materia o tema, una nueva forma de abordarla en el curso, planificar y crear modelos de trabajo académicos diferentes. Cambiar la forma de evaluación del tema, no puedo aplicar una técnica activa si luego continuo con el ciclo normal de calificación con pruebas escritas, debo adaptar el método del aprendizaje y buscar que el sistema evaluativo sea acorde a ese sistema.

- Entender que las técnicas STEM o activas no son una moda, sino una nueva forma de pensar y crear conocimiento constructivo y crítico en el proceso de enseñanza, es permanente, generará grandes esfuerzos en su aplicación, pero traerá la recompensa de preparar alumnos calificados en conocimientos científicos y técnicos, empoderados con las suficientes habilidades blandas para enfrentar el mundo futuro más veloz e incierto.

Existe mucha información sobre el cambio del aula tradicional al aula activa, no es nuestro objeto de análisis proponer las diferentes técnicas al respecto, pero si es objetivo de este artículo proponer un ejemplo, que ya ha sido realizado en el aula que busca adaptar la enseñanza de un tema contable del método tradicional a la técnica activa. Este cambio busca por tanto entregar al alumno una nueva ruta de su conocimiento, en forma ordenada, coordinada e integrada.

Abordemos el caso concreto de un tema en un curso de contabilidad, utilizaremos las formas abordadas por la Universidad de Laspau, SINAES, Programa para Innovar la Enseñanza y Aprendizaje en las Ciencias Sociales y Humanidades en Costa Rica [6], en la cual el autor y otros compañeros de la Universidad hemos participado. 
Planteamos el siguiente desarrollo metodológico:

1. Curso: Contabilidad IV.

2. Tema Contable: El flujo de Efectivo.

3. Competencia a Desarrollar: Capacidad del estudiante de analizar dos Estados Financieros (Balance General último año y anterior) y preparar conforme a la metodología propuesta por los autores utilizados, el Flujo de Efectivo.

4. Herramientas y recursos:

a. Lectura del Capítulo 14, libro Contabilidad Financiera de Guajardo. [7]

b. Lectura del Capítulo 1, Aplicaciones Prácticas de las NIIF, Vernor Mesén.

5. Método de casos: Solucionar los casos de Cenimsa y TV del Sureste, libro Contabilidad Financiera de Guajardo.

6. Identificar la posición de la taxonomía de Bloom como forma de determinar los énfasis del caso. Esto ayuda a determinar la técnica activa escogida y el sistema evaluativo. Siendo en nuestro caso, el criterio de aplicar el conocimiento teórico a la solución del caso, el objetivo más importante.

\section{TAXONOMÍA DE BLOOM APLICADA AL EJERCICIO DEL APRENDIZAJE AUTÉNTICO}

\begin{tabular}{|c|c|c|}
\hline INDICADOR & RÚBRICA & APLICACIÓN RÚBRICA \\
\hline Recordar & $5 \%$ & $\begin{array}{c}\text { Aplicación de concepto del tema } \\
\text { teórico }\end{array}$ \\
\hline Entender & $20 \%$ & $\begin{array}{c}\text { Encontrar el eje central del } \\
\text { manejo de efectivo en el aula }\end{array}$ \\
\hline Aplicar & $40 \%$ & $\begin{array}{c}\text { Desarrollar dos casos y cuadrar } \\
\text { los mismos con el flujo de } \\
\text { efectivo }\end{array}$ \\
\hline Analizar & $20 \%$ & $\begin{array}{c}\text { ¿Para qué sirven los datos de un } \\
\text { flujo de efectivo? }\end{array}$ \\
\hline Evaluar & $0 \%$ & $\begin{array}{l}\text { No es necesario en nuestro } \\
\text { esquema }\end{array}$ \\
\hline Crear & $15 \%$ & $\begin{array}{l}\text { Usar el excel para crear una } \\
\text { plantilla modelo }\end{array}$ \\
\hline TOTAL & $100 \%$ & \\
\hline
\end{tabular}

7. Encontrar o redactar la situación auténtica: El objetivo es que el estudiante descubra por sí mismo, bajo la guía de un autor y la del profesor como se prepara el modelo de un flujo de efectivo de una empresa. En este punto el énfasis de la evaluación autentica estará en la posibilidad de aplicar el modelo con dos ejercicios indicados y analizar los resultados de los mismos. Dicha aplicación deberá hacerse en una hoja de Excel donde se construirán ambos casos bajo el modelo propuesto por el autor.
8. Redactar las instrucciones y determinar las responsabilidades de los estudiantes por esta tarea.

\begin{tabular}{l|l}
\hline CURSO & Contabilidad IV \\
\hline MATERIA & $\begin{array}{l}\text { Preparación del Flujo de } \\
\text { Efectivo }\end{array}$ \\
\hline MATERIAL BASE & $\begin{array}{l}\text { Capítulo 14 Libro Contabilidad } \\
\text { Financiera de Guajardo. } \\
\text { Video como preparar el Flujo de } \\
\text { Efectivo }\end{array}$ \\
\hline EVALUACIÓN & $\begin{array}{l}\text { Se toma como un 20\% de la } \\
\text { nota total como trabajo de } \\
\text { investigación }\end{array}$ \\
\hline
\end{tabular}

9. Decidir cuál será la evidencia:

EVIDENCIAS: $\quad$ a. Lectura del Capítulo 14

Presentar un resumen ejecutivo del Capítulo.

b. Realizar dos casos prácticos:

Cenimsa

Televisora Sureste.

Presentar la solución de ambos casos

10. Prever el acompañamiento y la manera de retroalimentar

\begin{tabular}{l|l|l}
\hline PERIODOS & Presentación formal & Semana 3 \\
\hline & Avance 1 & Semana 8 \\
\cline { 2 - 3 } & Avance 2 & Semana 10 \\
\cline { 2 - 3 } & Entrega Proyecto & Semana 12 \\
\cline { 2 - 3 } & Revisión Materia & Semana 12 y 13 \\
\cline { 2 - 3 } & $\begin{array}{l}\text { Calificación y } \\
\text { aprovechamiento }\end{array}$ & Semana 14 \\
\hline
\end{tabular}

11. Montar el sistema de rúbrica de evaluación conforme a la taxonomía elegida, buscar el énfasis adecuado entre todas las actividades propuestas.

12. Realizar un ejercicio post-trabajo que permita indicar las falencias y las áreas de oportunidad de mejora. Este ejercicio utiliza las mismas técnicas que los elaborados por ellos bajo la conducción del profesor.

13. Dar la oportunidad al estudiante de volver a sus casos a corregir y mejorar las falencias.

14. Dar un cierre final del trabajo activo recalcando los valores aprendidos, los fines logrados y la evaluación obtenida por su participación. 


\section{Conclusiones.}

Es indudable que la complejidad, globalización y exigencias del mundo de los negocios de hoy en día hace que el sistema contable sea cada vez más dinámico y exigente.

Nos hemos movido de prácticas tradicionales de registro de la materialidad en papel hacia esquemas de acumulación electrónico, lo que permite al Contador centrar su énfasis de acción en el control de las operaciones y fuentes de datos, con el fin de garantizar la calidad e integridad de estos. El reto ante un mundo cada día más lleno de información será el preparar a estudiantes, hacia la visión crítica de los datos, su selección, validación, y preparación de modelos de análisis que favorezcan la tomas de decisiones empresariales.

Es imperativo dentro del aula la adopción de técnicas activas que permitan al estudiante a exponerse en un laboratorio de aprendizaje donde el pensamiento crítico y el empoderamiento académico le permitan integrar la técnica contable, la aplicación de las normas internacionales y el análisis de la información contable y financiera.

En nuestro caso presentado los resultados del ejercicio activo en comparación con el modelo tradicional ha favorecido la inteligencia técnicas de los estudiantes, la capacidad de resolver casos, el trabajo el equipo y el involucramiento en la materia. Los resultados evaluativos mejoran importantemente, pero más que eso, queda la satisfacción académica de haber empoderado a los estudiantes en el tema, haberlos hecho propietarios de su propio conocimiento, y haber permito hacer recomendaciones, cambios y mejoras constructivamente.

Solo de esta manera podemos avanzar hacia el conocimiento de nuevas formas de realizar transacciones, en un horizonte donde el cambio se ve en forma acelerada e impactando directamente a las empresas y la gente común.

Veremos llegar una fuerte época de aparición de nuevas formas de comercio, como el comercio electrónico, nuevas formas de pago como las monedas electrónicas y las "criptomonedas", veremos la llegada de la Big-Data como medio de conocimiento de las tendencias, la inteligencia artificial y muchos otros cambios más que impactarán en forma definitiva las prácticas de nuestros contadores en sus empresas.

La historia ha demostrado que la Contabilidad evoluciona a la par de la humanidad y se ha adaptado a las nuevas necesidades que vayan surgiendo en este cambiante mundo, pero esta herramienta tan necesaria e imprescindible está tan arraigada en nuestras empresas y organizaciones que jamás desaparecerá.

Estamos llamados si bien no a predecir el futuro, sí a preparar a los hombres y mujeres del mañana que tendrán en su mano el poder de decisión sobre este cambiante entorno, solo así podremos fundamentar nuestro quehacer profesional en la academia de nuestra Universidad cuando nuestros estudiantes puedan actuar y evolucionar hacia su pensamiento crítico.
La tarea no es fácil, pero la invitación al cambio está hecha, el trabajo grupal nos llama y la oportunidad de mejorar nuestro entorno educativo es más que una promesa una obligación.

\section{Referencias}

[1] IASB, International Accounting Standards Board, (2019), NIIF, Normas Internacionales de Información Financiera (NIIF), (En inglés como IFRS (International Financial Reporting Standards), Londres, Reino Unido.

[2] Dirección General De Tributación, Ministerio de Hacienda, (2018), DGT-R-029-2018, Criterios Interpretativos sobre la Aplicación de las Normas Internacionales De Información Financiera Respecto a la Normativa Tributaria.

[3] Asamblea Legislativa de la República de Costa Rica, (2018), Decreto Legislativo $N^{\circ}$ 9635, Fortalecimiento De Las Finanzas Públicas.

[4] Lic. Dorys Jackeline López Duarte, (2019) Consultor Internacional Asociación Interamericana de Contabilidad (AIC), Boletín Electrónico No. 111, 15 de Octubre de 2019.

[5] Vernor Mesén, (2017), Aplicaciones Prácticas de las NIIF completas, San José, Costa Rica.

[6] SINAES (Sistema Nacional de Acreditación Educación Superior) y Laspau (Afiliado a Harvard University), (2019), Programa de Capacitación Metodologías para el Aprendizaje Activo en las Ciencias Sociales y Humanidades en las Universidades de Costa Rica, San José, Costa Rica.

[7] Gerardo Guajardo Cantú, (2018), Contabilidad Financiera, México, $7^{\circ}$ Edición. 